\title{
Complejidad, instituciones y antropología Reflexiones sobre los límites del conocimiento antropológico en la "sociedad del conocimiento"
}

\author{
Oscar González Seguí
}

Los estudios de complejidad sirven como ejemplo para explorar el condicionamiento institucional e ideológico que marca los límites al conocimiento antropológico. Aquí se examina la relación de la antropología sociocultural con otros campos científicos, condicionada por la tradición, la formación profesional y la regulación estatal de la actividad cientíica. Se explora un marco de antropología del conocimiento y resalta la importancia de reflexionar sobre los límites al desarrollo disciplinario y sus causas concurrentes.

PALABRAS CLAVE: antropología, complejidad, conocimiento científico, instituciones científicas

\begin{abstract}
Complexity, Institutions and Anthropology. Reflections on the Limits of Knowledge in the "Knowledge Society"

Complexity Studies are a useful example for exploring the institutional and ideological conditioning that sets the limits to anthropological knowledge.This paper examines the relationship between Sociocultural Anthropology and other scientific fields, conditioned by tradition, professional formation, and the State's regulation of scientific activity. It also explores a framework of Anthropology of Knowledge and highlights the importance of pondering about the limits of disciplinary development and its concurrent causes.
\end{abstract}

KEY WORDS: anthropology, complexity, scientific knowledge, scientific institutions

OSCAR GONZÁlez Seguí: El Colegio de Michoacán, México. ogos@colmich.edu.mx 


\section{INTRODUCCIÓN}

$\mathrm{E}$ 1 conocimiento en antropología está limitado por varias razones: externas e internas, antiguas y novedosas, éticas y pragmáticas. Aquí quiero poner énfasis en los condicionamientos presentes que nos desconectan de una dinámica de búsqueda de saber nuevo, un objetivo que algunos quisiéramos mantener en primer término.

En este ensayo tomo como ejemplo de la disponibilidad de conocimientos la oferta de modelos de complejidad, un campo que se ha desarrollado durante más de veinte años y que podría tener incidencia en los estudios antropológicos. La disponibilidad de conocimiento no es suficiente para motivarnos a explorar más allá de los límites presentes, ya que otros factores influyen sobre la posibilidad de cambio.

Para indagar sobre esos límites, el marco será el de una "antropología del conocimiento" desde la cual intento reflexionar sobre la paradoja de que la antropología es, por una parte, una ciencia conocedora y crítica de las formas que, en paralelo, también sufre restricciones culturales y políticas que provienen de la sociedad y de las tradiciones académicas. La posibilidad de que, en la época en que se anuncia una "sociedad del conocimiento", brindemos un panorama histórico y cultural amplio de nuestra propia sociedad se ve frustrada en la práctica por condiciones (sociohistóricas, sin duda) que congelan los contenidos, métodos e instituciones del conocimiento sociocultural.

En el artículo analizo varios condicionamientos de la disciplina antropológica considerada como una ciencia que estudia los grupos y procesos humanos y su devenir. En tal sentido, los avances de los estudios de complejidad durante los últimos veinte años abren la perspectiva de estudiar relaciones y fenómenos emergentes en una sociedad muy interconectada ("globalizada") por las tecnologías, con creciente competencia de familias y personas por espacio, dinero, empleo, servicios, etcétera.

Este ensayo muestra que diversos modelos basados en la complejidad están disponibles para emplearse en antropología; destaca que también es tema nuestro una antropología del conocimiento (de la que somos juez y parte). También señala la existencia de diversos factores que ponen vallas a la relación de la antropología con el resto del conocimiento científico. Finalmente, aboga en favor de una interdisciplina capaz de entender problemas importantes de este momento histórico, que aporte conocimiento para identificar oportunidades de cambio social posible.

\section{LOS ESTUDIOS DE COMPLEJIDAD, UN CONOCIMIENTO DISPONIBLE}

Hace poco tiempo, en una revisión (González Seguí, en prensa) reflexionamos acerca de que en la antropología y las ciencias sociales el acercamiento al tema de la "complejidad" se hace desde varios puntos de vista. Éstos son: 1) la aplicación de herramientas metodológicas generales sobre desarrollos emergentes que parten de la similitud de configuraciones en los problemas planteados (redes, algoritmos genéticos y otros modelos complejos, cf. Reynoso, 2006); 2) los marcos "complejos" para estudios determinados (por ejemplo, García, 1986; García et al., 1988a, 1988b; Portugali, 1999); 3) desarrollos teóricos nuevos, que serían específicos para las ciencias sociales (por ejemplo, Huberman y Glance, 1993; Heylighen y Campbell, 1995); 4) la búsqueda de una comprensión nueva basada en la generalización de las ideas de emergencia de un orden en sistemas complejos, ya que la multicausalidad, la indeterminación, los saltos cualitativos y el orden emergente estarían enfrentados a modelos causales y a "la ciencia mecanicista". Nuevas formas de pensamiento y comprensión basadas en la complejidad ya estarían disponibles. Algunos autores citados con frecuencia que siguen esta línea son: Prigogine (1996), Prigogine y Stengers (1983), Maturana y Varela (1996), Capra (1996), Morin (1981-1992 y 2003).

Dados los múltiples puntos de vista, aquella revisión sobre complejidad y antropología llegaba a preguntarse acerca del efecto que las muchas ofertas de estudios sobre complejidad tendrían en la antropología. "La complejidad, como idea general, parece no tener detractores", se planteaba al principio, pero la conclusión sostenía que era dudoso que las distintas corrientes de las ciencias socia- 


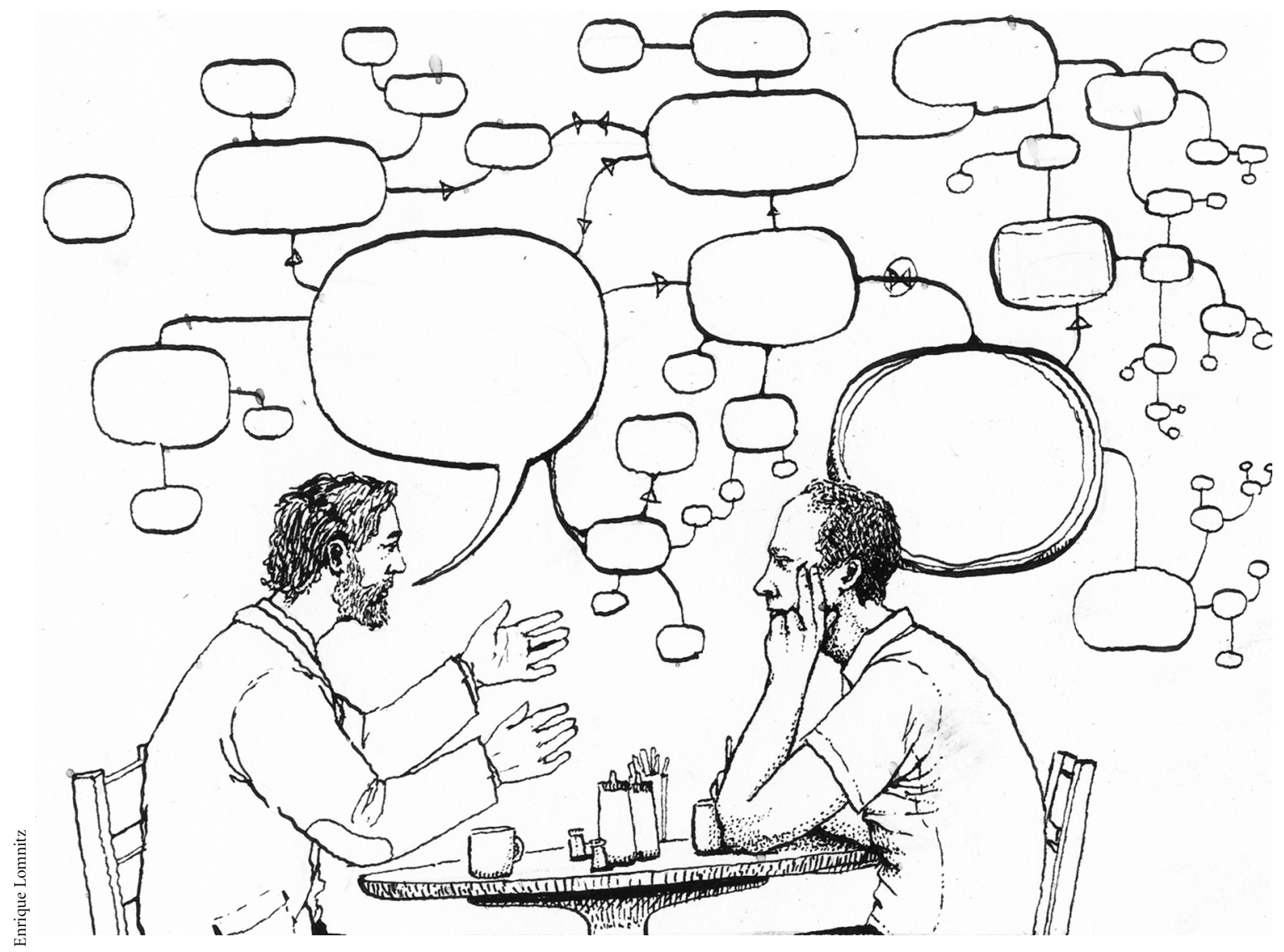

les acabaran unidas bajo un paradigma consensuado y mayoritario "de complejidad".

En el presente artículo trato de la conformación del temario y de la agenda de investigación en antropología a partir de la revisión de la propuesta de Fredrik Barth sobre antropología del conocimiento (Barth, 2002). Tomo como punto de partida la oferta de propuestas "complejas" que llega al público de las ciencias sociales. Para ello, comienzo por revisar sucintamente los modelos de complejidad que están disponibles.

Los modelos de complejidad ofrecen la posibilidad de una vía transdisciplinaria en las ciencias naturales y humanas. Su asunto principal y su base empírica son los procesos en los que se producen nuevas entidades e interacciones a partir de componentes y relaciones más simples. Los ejemplos son múltiples: material genético hecho de moléculas, organismos multicelulares, oscilaciones fisicoquímicas, sistemas inmunes, lenguajes, inteligencia, especialización de zonas cerebrales, transporte y ordenamientos urbanos y muchos otros fenómenos. En el nivel social sirven de ejemplo los procesos tecnológicos y el surgimiento de conocimiento, la aparición de nuevos niveles de integración sociopolítica, los estilos cerámicos, el crecimiento de las ciudades, las formas musicales, etc. (cf. Gell-Man, 1994; Horgan, 1995; Holland, 1995; Kauffman, 1993 y 1995; Lansing, 2003; Thrift, 1999; Johnson, 2003; Reynoso, 2006).

Las reflexiones sobre los cambios emergentes — de cantidad transformada en calidad, de combinación de partes que resultan en un todo diferente- son muy antiguas, pero en el siglo XX se desarrollaron propuestas teóricas basadas en observación empírica y creación matemática 
sobre dinámicas no lineales y sistemas complejos. Los ejemplos más sencillos de emergencia de orden son las oscilaciones de sistemas físicos simples — como los relojes de péndulo- y la conjunción de modelos matemáticos con observaciones y medidas realizadas por meteorólogos y biólogos, que también han servido de base para el desarrollo del campo científico de la complejidad. A esto hay que sumar que, en las últimas décadas, se ha invertido mucho trabajo creativo y recursos técnicos en el área de computación. Los resultados están ahora presentes en muchas herramientas de procesamiento de información y modelos aptos para investigar sistemas complejos, más allá de que las referencias empíricas sean mercados, ciudades, sistemas neuronales u osciladores electromagnéticos.

Las ciencias humanas, incluyendo la antropología, estudian muchos fenómenos que pueden ser caracterizados como complejos, tales como la misma emergencia de la especie, la(s) cultura(s) humana(s), los ordenamientos sociales de todo nivel, los mercados, las tecnologías, los lenguajes y sistemas semióticos, las formas los servicios urbanos, los sistemas de conocimiento, el manejo del medio ambiente. Las nuevas herramientas son, en principio, aplicables a los objetos de estudio de las ciencias humanas y a la formulación de sus problemas. Los modelos complejos son utilizados para entender, por ejemplo, la propagación de epidemias, los sistemas de tránsito, la evolución de formas de diseño (Eglash, 1999), la cooperación cultural (Reynolds, 1999), el funcionamiento de ciudades (Portugali, 1999) o los sistemas biosociales históricos estudiados por los arqueólogos (Gumerman y Gell-Mann, 1994; Marcus y Flannery, 1996).

El tema de la complejidad llega hasta los científicos sociales por textos de divulgación y publicaciones científicas. "Complejidad" (junto con caos, catástrofe, emergencia, etc.) es una palabra llamativa para la prensa y los medios. Nigel Thrift (1999) señala que las ideas sobre complejidad nos llegan por varias vías: 1) revistas y libros especializados que dan cuenta de los usos científicos de los modelos matemáticos y de computación, como los que se usan para entender los terremotos o los huracanes, o

para diseñar maquinarias, servicios urbanos o mecanismos para (intentar) ordenar el tránsito, etc; 2) literatura de autoayuda y similares, y también de humanidades, en las que la "complejidad" sería una forma nueva de superar limitaciones del conocimiento y prácticas que actualmente tenemos; 3) varios otros usos mediáticos y publicitarios de este término, incluyendo evangelios complejos más o menos asociados con la mercadotecnia (Thrift, 1999; Reynoso, 2006).

En México los paradigmas de la complejidad han estado presentes, con una divulgación y uso moderados, desde hace dos décadas. En la Universidad Nacional Autónoma de México (UNAM) hay varios institutos que aplican modelos complejos en sus investigaciones. Desde 1990 existe en el Instituto de Física un Departamento de Sistemas Complejos. El grupo más consolidado ha trabajado durante más de veinte años en dinámica de los sistemas complejos; investigan sobre biología teórica, dinámicas no lineales, biocomplejidad, sistemas complejos y ecología, y sociofísica (Miramontes, 1999; Boyer y Miramontes, 2003; Boyer et al., 2004, 2005; Cocho, 1999; Ramos-Fernández et al., 2004).

Podemos recapitular algunos antecedentes de estudios más o menos cercanos al tema de la complejidad en las ciencias sociales: en el Centro de Investigaciones Interdisciplinarias en Ciencias y Humanidades de la UNAM (CEIICH) se desarrollan investigaciones, presentaciones regulares y congresos anuales sobre complejidad. Uno de sus impulsores es Rolando García, quien fue innovador en los estudios multidisciplinarios emprendidos por el Centro de Investigación y de Estudios Avanzados (CINVESTAV) del Instituto Politécnico Nacional (IPN) en la Comarca Lagunera y la región del Bajío (García et al., 1988a y 1988b). Estas experiencias extendieron las posibilidades de contactos interdisciplinarios entre las ciencias sociales y naturales, así como con disciplinas tecnológicas y ambientales (García, 1994). En el CEIICH, donde García investiga hasta el presente, se realizan trabajos con modelos matemáticos y se exploran distintas posibilidades de interdisciplinas (García, 2006; Mansilla y Ruelas, 2005), además de la investigación epistemológica en ciencia social en la que sobresalen Pablo González Casanova y Rolando García (cf. González Casanova, 2004; García, 2000). 
En antropología, en el Instituto de Investigaciones Antropológicas de la UNAM hay una línea de investigación que contempla el tema de la complejidad y que se interesa en la memoria, las pervivencias culturales y la cognición, con base en trabajos etnográficos sobre distintas etnias de México (Pérez-Taylor, 2002, 2006).

Otra institución de antropología que tiene relación con las teorías de sistemas y temas antecesores de los estudios de complejidad en el presente es la Universidad Autónoma Metropolitana (UAM)-Iztapalapa. En el Departamento de Antropología se realizaron los trabajos de Roberto Varela (1984) y Leonardo Tyrtania (1999), que fueron investigaciones heterodoxas sobre antropología política y ecología cultural, inspiradas por Richard N. Adams, de la Universidad de Texas en Austin (en la que también residió Ilya Prigogine y fue profesor invitado Ángel Palerm). Adams se interesó en las estructuras disipativas para desarrollar su teoría del poder social basada en el manejo de energía (Adams, 1983 [1975]) y fue investigador visitante del Centro de Investigaciones Superiores del Instituto Nacional de Antropología e Historia (CIS-INAH), ahora Centro de Investigación y Estudios Superiores en Antropología Social (CIESAS), donde escribió su primer ensayo sobre el sistema humano como estructura disipativa (concepto de Prigogine) (Adams, 1978), que amplió más tarde en otro libro (Adams, 1988). Más recientemente, en el CIESAS se han realizado algunos trabajos que se acercan a temas de complejidad con interés moderado (Coronado, 2001; Molina, 2005). En otros ámbitos académicos, la Universidad Autónoma de la Ciudad de México (UACM) creó en 2002 una maestría en dinámica no lineal y sistemas complejos, y en El Colegio de San Luis, Aguilera Ontiveros y López Paredes (2001) trabajaron en la "construcción de modelos sociales basados en agentes” (cf. González Seguí, 2003).

Los nuevos modelos sirven para replantear problemas olvidados como la inferencia de procesos de difusión de todo tipo, los mecanismos de interfase entre fenómenos ecológicos y económicos, o la existencia de patrones culturales que subyacen a los diseños. La complejidad no sólo es una herramienta novedosa, sino que lleva implícita la posibilidad de captar y plantear problemas distintos de los que surgen de los paradigmas político-intelectua- les dominantes. En América Latina han aparecido recientemente varios libros dedicados a modelos y temas de complejidad, que ayudan a comprender su relevancia para las ciencias sociales, en particular para la antropología: Reynoso, 2006; García, 2006; Pérez-Taylor, 2002 y 2006; Grupo Antropocaos, 2006 y, en la sociología de Cuba, Sotolongo y Delgado, 2006.

Los estudios sobre complejidad ya no son tan novedosos, y los trabajos que acabamos de citar no son suficientes para desmentir que la receptividad hacia esas propuestas es escasa. Necesitamos explorar más el tema del conocimiento en las instituciones.

\section{VICISITUDES DE LA PRODUCCIÓN DE CONOCIMIENTO EN LAS INSTITUCIONES}

Para la antropología, el conocimiento es más que un tema de estudio. A esta disciplina le atañen los cambios, la distribución social, los agentes y procesos de transmisión, el mantenimiento o extinción de conocimientos en grupos y los tiempos sociales. Además, al ser la antropología misma una actividad de conocimiento, la práctica de los antropólogos es un campo de su interés, que incluye la selección, permanencia e influencias mutuas entre el conocimiento "antropológico" y el medio social en que existe la antropología. Ésta fue la preocupación de Fredrik Barth en una conferencia magistral dirigida en especial a sus colegas, en la que destacó el escaso desarrollo de una antropología del conocimiento y consideró como base su propia investigación en Nueva Guinea y Bali. Con la intención de fundar un método antropológico para el estudio del conocimiento humano situado en grupos e instituciones, destacaba que la organización del conocimiento no sólo tiene "un corpus" de contenidos, sino que éste es sólo un componente. El conocimiento tiene tres aspectos cruciales e interrelacionados:

Primero, cualquier tradición de conocimiento contiene un corpus de aserciones sustantivas e ideas sobre aspectos del mundo. Segundo, ese corpus debe ser proporcionado y comunicado en uno o varios medios como serie parcial de representaciones, como palabras, símbolos concretos, gestos, acciones. Y tercero, será distribuido, comunicado, em- 
pleado y transmitido dentro de una serie de relaciones sociales instituidas. Estas tres caras del conocimiento están interconectadas $[\ldots]$ mi tesis es que estas tres caras del conocimiento aparecen juntas precisamente en las particularidades de la acción en cada evento de aplicación del conocimiento, en cada transacción de conocimiento, en cada performance (Barth, 2002: 3, trad. propia).

Esta conferencia del profesor Barth viene al caso que trato de indagar en estas páginas, ya que me interesan los limites del y rechazos al corpus de conocimiento antropológico social actual en relación con los estudios sobre complejidad. Así, aceptando su propuesta, partiremos de la referencia a las instituciones.

Para completar un marco de referencia, podemos agregar las reflexiones antropológicas de Mary Douglas sobre el condicionamiento social de los olvidos, las postergaciones y los rechazos a ciertos temas de investigación y a la ciencia ${ }^{1}$. En una serie de conferencias titulada Cómo piensan las instituciones (Douglas, 1986), Douglas destacaba el retraso, el olvido y el rescate de conocimientos en concordancia con las condiciones institucionales predominantes y resaltaba la importancia de que conozcamos cómo las instituciones, que no tienen mente propia, influyen en las nuestras:

No se asombren de que ellas nos recluten fácilmente dentro de su autocontemplación narcisista. Cualesquiera problemas que tratemos de pensar son transformados automáticamente en sus propios problemas organizacionales. Las soluciones que profieren sólo vienen del limitado rango de su experiencia. [...] Para nosotros, la esperanza de una independencia intelectual es resistir, y el primer paso necesario para la resistencia es descubrir cómo se establece un agarre institucional sobre nuestras mentes (Douglas, 1986: 92; trad. propia).

A partir de las reflexiones de Barth y Douglas, quiero enfocarme en el cambio que ha sobrevenido en la comunicación de la disciplina antropológica, tanto internamente como con el resto del conocimiento científico

\footnotetext{
${ }^{1}$ Otras disciplinas científicas y tecnológicas registran ejemplos varios, como la economía ecológica (Martínez Alier y Schlüpmann, 1991), postergada durante un siglo, o el retraso de la aparición pública de la teoría de la evolución de Darwin (Kitcher, 2001).
}

contemporáneo, ya que hasta la década de 1970 la profesión antropológica fue variada en sus intereses, que podían versar en asuntos como fósiles, modas, lenguas, religiones y técnicas. Pero después hubo cambios en sus temas de investigación y en sus instituciones, en un desplazamiento que es más complejo (uso adrede la palabra) que la narración que hacemos en los cursos introductorios de historia de la antropología, según la cual la insuficiencia de las explicaciones de distintas escuelas es superada por enfoques posteriores en el tiempo.

Según el marco de comprensión que nos ofrece Fredrik Barth, el conocimiento, en este caso antropológico, consta de un corpus, medios e instituciones. La pregunta de ahora es en qué medida el estado actual de las instituciones y los métodos permite explorar contenidos y herramientas nuevos o atípicos. Siguiendo también a Mary Douglas, parto de que es necesario comprender los condicionamientos institucionales sobre "las mentes" de los conocedores (en este caso los antropólogos).

Es importante, entonces, considerar los cambios en la organización y la política de las instituciones de ciencia y tecnología de México (y otros países latinoamericanos)². El tema es inmediato, ya que en los últimos años la práctica de producción de conocimientos científicos ha estado fuertemente influida por políticas enfocadas en la vinculación y las demandas empresariales o de otros sectores. Lo expresa, precisamente, un antropólogo preocupado por el tema en relación con la historia de su institución:

[...] las formas de creatividad científica y humanística son tratadas como servicios para consumo que giran en torno a un sistema de oferta y demanda, este "efecto multiplicador" [que antes fuera el objetivo institucional] será sustituido por un sistema de estímulos que no estimula las condiciones que posibilitan la apropiación de conocimiento y la producción creativa, sino que estimula una tasa creciente de producción incapaz de distinguir entre el credencialismo y la educación; es decir, un sistema de evaluación fácilmente comprometido con la impunidad y la simulación (Roth, 2008).

\footnotetext{
${ }^{2}$ Los países de América Latina se parecen, una vez más, en la medida en que reciben directivas de las mismas instituciones de regulación internacional (Banco Mundial, Organización para la Cooperación y el Desarrollo Económico [OCDE], etc.).
} 
Esta reflexión se suma a las preocupaciones manifestadas por otros investigadores. Eduardo Ibarra Colado destaca los sistemas de evaluación del trabajo en las instituciones académicas. Los académicos e investigadores científicos son evaluados, desde la década de 1990 en México y otros países, mediante una contabilidad de la "producción” (publicaciones, cursos, graduados) que no admite desvíos individuales, con calendarios que consideran y premian el número de "productos" y el "justo a tiempo" de la productividad, a través de procedimientos que devaluaron el sentido de producción de conocimiento nuevo y la reproducción de las habilidades. Estas prácticas marcan hoy a todas las organizaciones al punto de hacerles perder aspectos de su identidad que se habían ido consolidando durante décadas (Ibarra Colado, 2000; Roth, 2008).

La mencionada conferencia de Fredrik Barth sobre antropología del conocimiento trató, además de los casos etnográficos, sobre la influencia de la institucionalidad neoliberal en la antropología del Reino Unido. Las universidades británicas están sometidas desde la década de 1990 (iigual que en México!) a auditorías de contabilidad pública y "evaluaciones de calidad", así como a competencias entre distintos programas por llegar a lo más alto de un ranking. "Lo que se pone en las manos de este Leviatán burocrático es nada menos que el poder de reemplazar y reconfigurar los criterios de validez que gobiernan el conocimiento antropológico en Gran Bretaña" (Barth, 2002: 9). Barth concluye con un llamado de atención para que los antropólogos cuiden su propio campo de conocimiento. Y éste no es el único investigador que se preocupa por el tema de la "cultura de auditoría" en la antropología británica (cf. Shore y Wright, 1999).

También en Estados Unidos existe inquietud ante las políticas que afectan el conocimiento en antropología. Una década atrás Anna Grimshaw y Keith Hart denunciaron una crisis de la antropología como resultado de la institucionalización profesional:

El problema de aconsejar sobre nuevas formas de indagación antropológica debe resolverse en el nivel de la práctica social, no de las ideas. En particular es tiempo para los antropólogos de repensar si es prudente haber comprometido de manera tan completa el futuro de su proyecto colectivo en manos de las instituciones de la burocracia académica (Grimshaw y Hart, 1994: 259, trad. propia).

Por lo tanto, no es muy novedoso comprobar que también entre nosotros las ciencias existen en un medio institucional que las condiciona. Sin embargo, la inserción institucional de las ciencias sociales de acuerdo con políticas que regulan en general "la ciencia, la tecnología y la innovación” es afectada por una ideología que enuncia cómo se organiza la sociedad y cuáles son sus motores y problemas. Las ciencias sociales, incluida la antropología, existen subordinadas a esa ideología que dicta a priori lo que debiera ser conocimiento producido mediante investigación.

\section{LA PERSISTENCIA DE "LAS DOS CULTURAS" Y LA FORMACIÓN EN LA ESCUELA MEDIA}

Otra influencia sobre la definición del conocimiento antropológico y sus maneras proviene de la tradición de las humanidades y de la separación del conocimiento en "dos culturas", un cisma originado en las universidades europeas de fines del siglo XVIII. La división fue así: por un lado, las ciencias naturales, a cargo del conocimiento sobre la naturaleza; por el otro, un movimiento antiiluminista al que se llamó "humanidades", que comprendía la filosofía, el lenguaje y las letras, la historia del arte, la musicología y la historia. Ya antes las ciencias naturales se habían separado del saber filosófico que incluía todo. Ciencias y filosofía comenzaron a funcionar separadas unas de la otra. Así lo resume el Informe Gulbenkian:

Fueron más bien los que no eran científicos naturales - historiadores, anticuarios, estudiosos de literaturas naturales- los que más hicieron por resucitar las universidades durante el siglo XIX, utilizándolas como mecanismo para obtener apoyo del Estado para sus trabajos eruditos. Ellos atrajeron a los filósofos naturales hacia las nacientes estructuras universitarias para beneficiarse del perfil positivo que éstas poseían, pero el resultado fue que desde entonces las universidades pasaron a ser la sede principal de la continua tensión entre las artes o humanidades y las ciencias, que ahora se definían como modos de conocimiento 
muy diferentes y para algunos antagónicos (Wallerstein, 1996: 10).

Respecto a lo que nos atañe, sólo queremos resaltar que somos herederos de antiguas pugnas provocadas por esta separación de las facultades de humanidades de las ciencias. Esta brecha ideológica se consolidó en el siglo XIX y la disputa ha sido denominada de "las dos culturas" y está todavía vigente, aunque hace ya medio siglo parecían dadas las condiciones de su superación mediante una "tercera cultura" (cf. Snow, 1964).

La antropología en sus primeras décadas (con variantes según las escuelas y los países) parecía ser pionera de esta tercera cultura, pues estaba conectada tanto con las humanidades como con las ciencias naturales. Sin embargo, en la década de 1960 la brecha entre las "dos culturas" cobró un nuevo impulso con cambios acaecidos en las universidades de Europa y Estados Unidos. La influencia de esos cambios ha sido notable en la antropología: "Trabajos en distintos campos de las ciencias sociales y las humanidades confluyeron bajo el rubro de 'estudios culturales' que llevaron a conclusiones tendientes a deslegitimar las premisas universalistas sobre las que se había construido la estructura relacional de las ciencias naturales, las ciencias sociales y las humanidades" (Lee, 2000: 790).

En un proceso que duró varias décadas, la antropología sociocultural fue desligándose de las otras tres ramas de la clasificación estadounidense de mediados del siglo XX (arqueología, lingüística y antropología biológica) hasta que dichas especialidades "básicas" desaparecieron de los planes de estudio de la antropología sociocultural. En la especialización reinante, los profesores y estudiantes se alejan de quienes antes fueran sus colegas. Además, una proporción importante de los profesionales de las ciencias sociales adhieren a posiciones denominadas "críticas", que fundan su rechazo a "las ciencias" por ser éstas un producto de "la modernidad" (cf. Knorr-Cetina, 1981; Latour, 2003). Según esta línea, el conocimiento científico debe entenderse de acuerdo con las motivaciones, las ideologías y las circunstancias de sus productores, de lo cual es inseparable. El conocimiento científico (así, en general) es entonces moralmente sospechoso y no califica como verdadero, ni siquiera de modo provisorio, salvo para los grupos que los generan. Estas ideas, con gran influencia en los medios antropológicos de las últimas décadas, frustran a priori no sólo un posible diálogo "de culturas", sino también los trabajos transdisciplinarios con [los practicantes y los conocimientos de] las ciencias naturales.

El rechazo a las matemáticas y ciencias naturales por parte de algunas corrientes de humanidades tiene consecuencias en otros niveles educativos en tanto da justificación a la formación incompleta de los estudiantes de nivel medio. En el bachillerato se ve reforzada una tradición anacrónica que clasifica a las carreras según las facultades y a los alumnos según supuestas vocaciones. Sobre esa base se sigue separando a los alumnos de "carreras humanísticas" de los clasificados en otros rubros (carreras biomédicas, ingeniería, etc. $)^{3}$. En consecuencia, los Conocimientos sobre ciencias naturales y matemáticas de los historiadores, lingüistas, arqueólogos, sociólogos, abogados, economistas, administradores, etc., permanecen en un nivel básico y los futuros antropólogos (entre otros) no disponen de elementos científicos ni siquiera propios de escuela preparatoria. Del mismo modo, los de la rama de ingeniería ignoran contenidos de historia o de literatura. La comunicación transdisciplinaria de los futuros profesionales y científicos se hace literalmente impensable por el completo desconocimiento de otros estilos, métodos y técnicas de indagación, formas de discusión y consenso, herramientas de lógica, modelación, criterios de validación, etc., que resultan en la imposibilidad de comunicación con "la otra cultura". Una brecha fundada en causas mucho más vulgares que los motivos epistemológicos a veces invocados predispone a la defensa profesional. La separación de formas de conocer y de reconocerse $-\mathrm{y}$ discriminarse entre especialistas de ciencias diferentes - termina en un perjuicio general para los participantes y para la causa del conocimiento social.

\footnotetext{
${ }^{3}$ Aunque la variación de programas es grande, en escuelas de Michoacán que estudiamos, los estudiantes de bachillerato se "orientan" en 2007 según tres ramas: humanidades, ciencias biomédicas y ciencias exactas e ingeniería. Los de "humanidades" no reciben clases de ecología o biología; los de "exactas e ingeniería" no llevan introducción al derecho o a la sociología.
} 


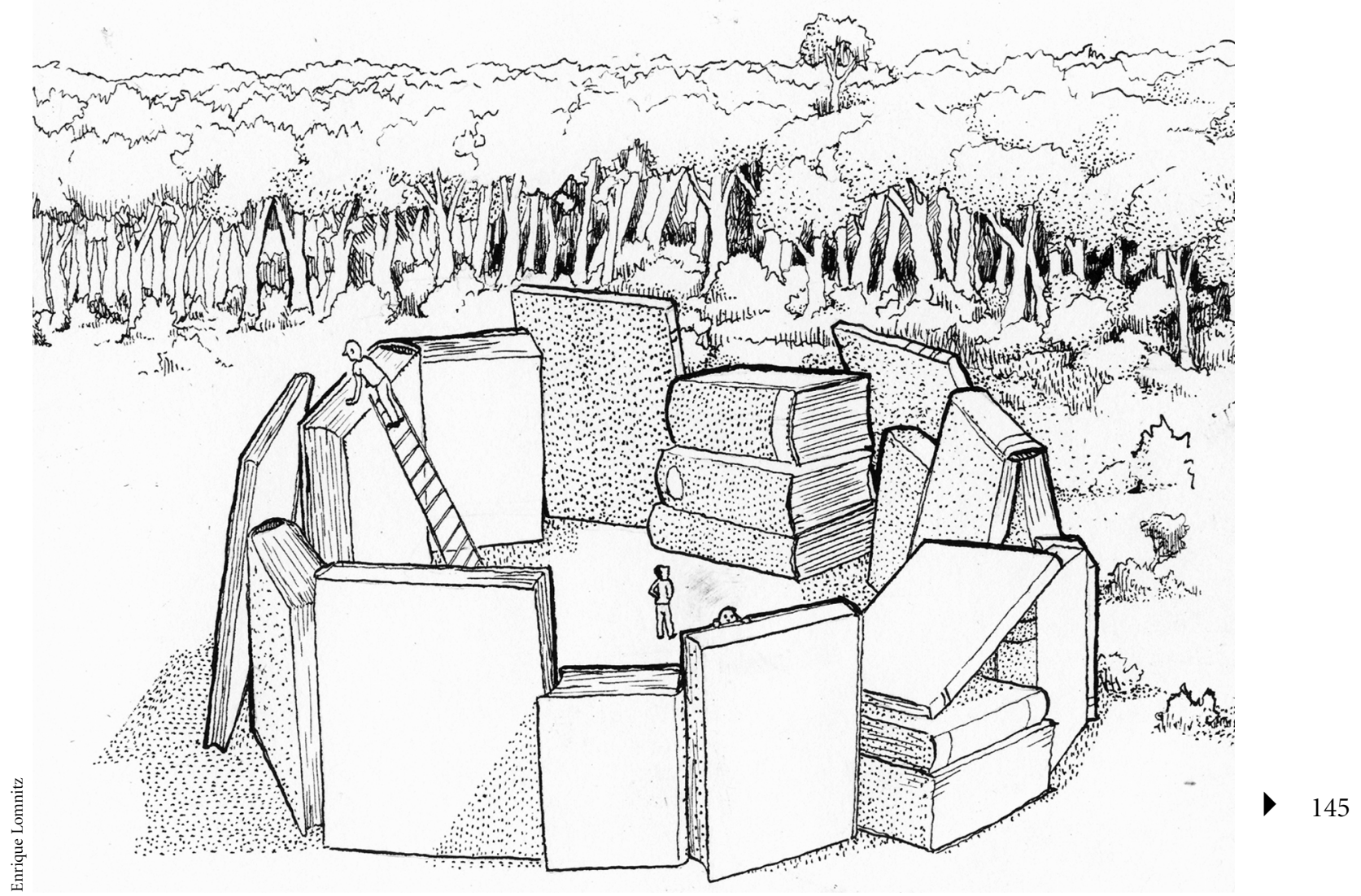

\section{CONDICIONAMIENTOSY FUTURO}

Los condicionamientos sociales sobre la producción, mantenimiento y fronteras del conocimiento antropológico ${ }^{4}$ son la primera clave para entender nuestra situación presente. Nuestro cuestionamiento es sobre el aislamiento de (buena parte de los practicantes de) la antropología social con respecto a las demás ciencias, en particular a otras ciencias que estudian aspectos humanos y temas de interés antropológico ${ }^{5}$.

\footnotetext{
${ }^{4}$ La reflexión sobre este punto puede aplicarse a otros conocimientos. Aquí deliberadamente no exponemos argumentos epistemológicos sobre la separación de ciencias sociales y ciencias naturales. ${ }^{5}$ Ejemplos de conocimientos que tomamos poco en cuenta, aun cuando aparecen en revistas de antropología como Annual Review of Anthropology, Current Anthropology, The Journal of R.I.A., son la psicología evolucionista (cf. Mithen, 2006; Berthenthal, 1996; Tooby y
}

Hemos puesto en el primer lugar de los factores limitantes la ya mencionada presión de las instituciones y destacado como subproducto de la misma "el control de nuestras mentes" que mencionaba Douglas. Por eso nos

Cosmides, 1997; Tomasello, 1999; Ehrlich y Feldman, 2003; Caporael, 2001; Jones, 1999) o la neurociencia social cognitiva (Gazzaniga, 2000; Cacioppo, 2002). En psicología social hay varias teorías con base en investigación (Burke, 2006). Incluyen "identidad" en el nivel grupal (Hogg, 2006) e individual (Stets, 2006). Otros asuntos son los estudios de interacción con computadoras (Suchman, 2006) o los temas sociológicos sobre adicción química (Smith y Stevens, 2002). La teoría de los polisistemas, formulada hace unos quince años por un investigador de literaturas nacionales, propone estudiar el sistema semiótico de la cultura (Even-Zohar, 2005). La contextualidad de la cultura es un tema recurrente que espera profundización interdisciplinaria con estudios de lingüística, psicología, sociología y de la misma antropología urbana (Hannerz, 1980). La construcción cultural es tema nuestro, pero la percepción intercultural no tanto (cf. Hong et al., 2000; Hacking, 2001). 
fijamos en que la política científica ajusta presupuestos, tiempos de informes y resulta en la adaptación hacia el interior y el exterior de las instituciones y las personas. La ciencia social (digo contenidos y prácticas de antropología, o de otra disciplina) se hace con actividades de investigación, docencia y uso del tiempo de la comunidad profesional. Los factores que la conforman son más las presiones y reacciones profesionales que la agencia, análisis y discusión colectivos. La tendencia predominante parece ser hacia la homogeneización de las instituciones, los temas y las personas, antes que a la diversidad que propugnamos en otros campos, que queda postergada en el nuestro ${ }^{6}$. Si el condicionamiento social es inevitable, la falta de discusión sobre el tema parece preocupante.

En este ensayo solamente podemos sugerir que las limitaciones debidas a las relaciones entre instituciones y docentes-investigadores-funcionarios del Estado en los entornos institucionales educativos y de investigación hacen que el conocimiento sea la última variable que ajusta el funcionamiento institucional (y esta reflexión puede ser válida más allá del campo que nos ocupa).

Anteriormente dejamos constancia de la existencia de algún interés por el tema de la "complejidad". No sabemos cuál puede ser la importancia futura de los modelos o paradigmas complejos, pero nuestro argumento apunta más a la necesidad de apertura hacia el conocimiento disponible que a la defensa de un modelo o metodología en particular

Parecía que la antropología, que un siglo atrás intentaba ser la "ciencia del hombre", le pondría relatividad - pero no menos rigor- a los conocimientos de la sociedad moderna. Tal era la intención de los investigadores en el Estrecho de Torres, en las Trobriand o en los trabajos de Evans-Pritchard en África, o cuando Edmund Leach trataba de comprender-proponer mecanismos sobre las transformaciones de una sociedad Kachim. Escuchamos y repetimos hasta el cansancio que

\footnotetext{
${ }^{6}$ La afirmación carece aquí de base empírica, pero un vistazo a los títulos de tesis y de artículos, o a los contenidos de las asignaturas deja la impresión de temáticas restringidas y repetidas, antes que de diversidad. Por supuesto, podrían usarse técnicas cienciométricas para documentarlo, pero no sé si se justifica el esfuerzo.
}

esa antropología era colonialista e imperialista (y sin duda surgía de instituciones del imperio). Los adjetivos no son suficientes para desechar todo el conocimiento de una época: muchas investigaciones no eran una justificación del colonialismo; profundizaron y desarrollaron conocimiento. La unidad biológica de la especie humana, por ejemplo, fue, y sigue siendo, una verdad de valor incalculable en la lucha contra la discriminación racial7.

Más recientemente en México, Ángel Palerm dedicó ingente esfuerzo por el desarrollo de una antropología con énfasis en el trabajo de campo y en la información histórica, y se interesó por emplear modelos que permitieran comprender los cambios históricos y las vías de transformación social. Estudió los sistemas de regadío, de organización del trabajo y de la circulación de mercancías, con exploración y crítica de modelos teóricos. Recordaba Eric Wolf que:

Ángel se tomaba la teoría en serio [...]. Durante los últimos años de su vida se esforzó denodadamente por transmitir a colegas y estudiantes la importancia de la construcción teórica en el pasado y para proporcionarles la capacidad de remodelar los entendimientos teóricos en el futuro (Wolf, 1986: 582).

Virginia García Acosta escribe también al respecto:

Ángel nos enseñó muchas cosas [...] entre ellas la importancia y el papel que en la investigación social deben jugar los modelos teóricos. Su carácter no puede ni debe ser siempre universal y definitivo, constituyen abstracciones de la realidad y deben ser susceptibles de modificarse (García Acosta, 2000: 234).

El interés de Palerm era "hacer una ciencia tan racionalmente transformadora de la sociedad como las de-

\footnotetext{
${ }^{7}$ Varias escuelas de antropología son desechadas sumariamente en cursos introductorios: preguntas sobre la evolución de las sociedades y la cultura quedan sepultadas con el evolucionismo; las de difusión de lenguajes, tecnologías, etc., con el difusionismo; los mecanismos socioculturales son funcionalistas y ya no pueden pensarse; la organización cognitiva común desaparece con el estructuralismo. Finalmente, la idea de que los sujetos humanos pueden tomar en sus manos el destino histórico de su sociedad también, porque es una idea marxista superada.
} 
más ciencias lo son ya de la naturaleza" (Palerm, 1980: 11, cit. por Palerm Viqueira, 2000: 97). La preocupación por probar modelos tiene un precursor destacado en la antropología mexicana.

He argumentado que nuestro descuido presente respecto al examen de nuevos recursos teórico-metodológicos de investigación se explica, principal aunque no exclusivamente, por el condicionamiento de la producción antropológica en entornos institucionales. Las posibilidades de probar modelos y conocimientos nuevos van a la par con la posibilidad de que el conocimiento antropológico pueda conformar un programa propio transdisciplinario para la época llamada de "la sociedad del conocimiento". Examinar los conocimientos y modelos nuevos tiene importancia, pero más la tiene todavía relacionar la tarea con la recuperación de aspiraciones abandonadas por una ciencia sociocultural humana que, en consonancia con el programa de Palerm, indague sobre el futuro social posible (para lo cual podrían aplicarse, $o$ no, las herramientas de la complejidad).

$\mathrm{Al}$ igual que en la práctica política, hace un siglo en la ciencia social se tenían objetivos deseables y misiones que hoy han sido abandonadas. Si otro mundo humano es posible, la antropología social debería (podría, puede) ser protagonista. Sin restar importancia a los trabajos del presente, cabe volver a proponer y analizar problemas que hemos dejado en el olvido, como la explotación del trabajo, o que ahora sólo se estudian excepcionalmente en antropología, como las organizaciones formales y profesionales, los niños y jóvenes, la economía ilegal, las "instituciones totales", los servicios del gobierno y los privatizados (de los que carecemos aún de descripciones etnográficas desde la visión de los usuarios).

Siguiendo las enseñanzas de los maestros, vimos que para conocer antropológicamente el conocimiento es necesario tomar en cuenta de manera conjunta las instituciones, los medios y los contenidos. Si identificamos medios y contenidos posibles, pero olvidados o ignorados, debemos preguntarnos por la causa institucional de esta ignorancia. La revisión de la dinámica del conocimiento en la antropología de cada país y, más específicamente, en cada institución es necesaria. Tenemos que entender las instituciones en que intentamos transmitir o desarrollar conocimiento antropológico (o de otra marca); entender la trama institución-medios-conocimiento, como enseñaba Barth. Entender la apropiación institucional de nuestras mentes, como sugería Douglas. Pero entender sería insuficiente y cambiar las reglas puede ser más necesario.

Para redondear la propuesta: no somos tan responsables ni tan inocentes del estado de nuestra disciplina. Los académicos tenemos alguna agencia en la selección de lecturas, ideas, contenidos y herramientas en nuestro ámbito de trabajo. Podemos influir, hasta cierto punto, en la organización social inmediata, y aun en las instituciones que regulan su actividad; sabemos manejar el lenguaje y contamos con medios para difundir mensajes. Los antropólogos compartimos — creo- aspectos éticos básicos: buscar nuevo conocimiento social; respetar la diversidad de las personas y grupos; ser críticos de la desigualdad y de las injusticias, y entender cómo se producen y persisten. Pero las intenciones y las posibilidades de una actividad intelectual que incida en la sociedad pueden quedar sepultadas en escritos y retórica.

"Otro mundo es posible" sostienen los movimientos altermundistas y algunos científicos sociales quieren apoyar esos movimientos. Para ello, las ciencias sociales tienen corrientes críticas que se refieren a acontecimientos futuros que pueden suceder, o a pasados que pudieron haber sucedido (Tilly, 1997: 17). En la dinámica de profesionalización, y en una sorda tensión con las tecnocracias, impedimos los encuentros con distintos conocimientos que podrían encaminarnos hacia utopías, a explorar de nuevo, con mayor urgencia que antes, las posibilidades de que la especie que hace historia la haga más conscientemente, como lo intentaban hace un siglo y medio los fundadores de las ciencias sociales. Los buscadores de utopía no se han extinguido y todavía algunos llegan a estudiar ciencias sociales (más que a buscar una profesión domesticada). "Otro mundo posible" requiere de parte nuestra la reelaboración que busque una ciencia humana abierta e interdisciplinaria; posible y que entienda posibilidades. El conocimiento puede llevar al cambio y, al revés, como también enseñaron los fundadores, el cambio al conocimiento. 


\section{Referencias}

Adams, Richard N., 1978, La red de la expansión humana, Centro de Investigaciones y Estudios Superiores en Antropología Social, México.

__ 1983 [1975], Energía y estructura. Una teoría del poder social, Fondo de Cultura Económica, México.

— 1988, The Eighth Day. Social Evolution as the Self Organization of Energy, University of Texas Press, Austin. [Trad. al español: El octavo día..., Universidad Autónoma Metropolitana, México, 2001]

Aguilera Ontiveros, Antonio y Adolfo López Paredes, 2001, Modelado multiagente de sistemas socioeconómicos. Una introducción al uso de la inteligencia artificial en la investigación social, El Colegio de San Luis, San Luis Potosí.

Barth, Fredrik, 2002, "An Anthropology of Knowledge”, Current Anthropology, vol. 43, núm. 1, febrero, pp. 1-18.

Bertenthal, Bennet I., 1996, "Origins and Early Development of Perception, Action, and Representation”, Annual Review of Psychology, núm. 47, pp. 431-459.

Boyer, D. y O. Miramontes, 2003, "Interface Motion and Pinning in Small-World Networks", Physical Review E, vol. 67, núm. 3, art. núm. 035102, parte 2, marzo.

_, G. Ramos-Fernandez, O. Miramontes, J. L. Mateos, G. Cocho y H. Ramos, 2004, "Deterministic Walks for Animal Foraging", taller "Transport and Self-organization in Complex Systems”, Porto Alegre, Brasil.

—, G. Ramos-Fernandez, O. Miramontes, J. L. Mateos, G. Cocho, H. Larralde, 2005, "Spontaneous Emergence of Groups in Levy-like Foraging Primates", taller "Stochasticity and Nonlinearity in Three Continents", 30 de marzo-1 ${ }^{\circ}$ de abril, Santa Fe, Nuevo México.

Burke, Peter (ed.), 2006, Contemporary Social Psychological Theories, Stanford University Press, Stanford, California.

Cacioppo, John T. (ed.), 2002, Foundations in Social Neurocience, Bradford Books.

Caporael, Linnda R., 2001, "Evolutionary Psychology: Toward a Unifying Theory and a Hybrid Science", Annual Review of Psychology, núm. 52, pp. 607-628.

Capra, Fritjof, 1996, The Web of Life. A New Scientific Understanding of Living Systems, Anchors Books, Nueva York.

Cocho, Germinal, 1999, “Teorías de sistemas: Haken, Prigogine, Atlan y el Instituto de Santa Fe”, en Santiago Ramírez (coord.), Perspectivas en las teorías de sistemas, Centro de Investigaciones Interdisciplinarias en Ciencias y Humanidades (CEIICH), Universidad Nacional Autónoma de México, Siglo XXI, México, pp. 45-50.

Coronado, Gabriela, 2001, "La identidad mexicana como sistema dinámico complejo”, ponencia, Congreso Internacional de Estudios Interdisciplinarios y Complejidad, Centro de Investigaciones Interdisciplinarias en Ciencias y Humanidades (CEIICH), Universidad Nacional Autónoma de México, 22-26 de octubre, México.

Douglas, Mary, 1986, How Institutions Think, Syracuse University Press, Syracuse.

Eglash, Ron, 1999, African Fractals. Modern Computing and Indigenous Design, Rutgers University Press, New Brunswick.

Ehrlich, Paul y Marcus Feldman, 2003, "Genes and Cultures. What Creates our Behavioral Phenome?", Current Anthropology, vol. 44, núm. 1, febrero, pp. 87-107.

Even-Zohar, Itamar, 2005, "Polysystem Theory (Revised)", Papers in Culture Research, en línea: < http://www.tau. ac.il/ itamarez/> (consultado en noviembre de 2007).

García, Rolando, 1986, "Conceptos básicos para el estudio de sistemas complejos”, en Enrique Leff (ed.), Los problemas del conocimiento y la perspectiva ambiental del desarrollo, Siglo XXI, México, pp. 45-71.

__ 1994, "Interdisciplinariedad y sistemas complejos", en Enrique Leff (comp.), Ciencias sociales y formación ambiental, Gedisa, Barcelona, pp. 85-124.

— 2000, El conocimiento en construcción: de las formulaciones de Jean Piaget a la teoría de sistemas complejos, Gedisa, Barcelona.

__, 2006, Sistemas complejos. Conceptos, métodos y fundamentación epistemológica de la investigación interdisciplinaria, Gedisa, Barcelona.

— et al., 1988a, Modernización en el agro. ¿Ventajas comparativas para quién? El caso de los cultivos comerciales en el Bajio, UNRIS, Institute for International Assistance and Solidarity (IFIAS), Centro de Investigación y Estudios Avanzados del Instituto Politécnico Nacional (Cinvestav), México.

— et al., 1988b, Deterioro ambiental y pobreza en la abundancia productiva. El caso de la Comarca Lagunera, IFIAS, Centro de Investigación y Estudios Avanzados del Instituto Politécnico Nacional (Cinvestav), México.

García Acosta, Virginia (coord.), 2000, La diversidad intelectual. Ángel Palerm, in memoriam, Centro de Investigaciones y Estudios Superiores en Antropología Social, México.

Gazzaniga, M. S. (comp.), 2000, The New Cognitive Neurosciences, MIT Press, Cambridge, Massachusetts.

Gell-Man, Murray, 1994, The Quark and the Jaguar. Adventures in the Simple and the Complex, Little Brown, Londres [hay traducción al español].

González Casanova, Pablo, 2004, Las nuevas ciencias y humanidades. De la academia a la política, Instituto de Investigaciones Sociales, Universidad Nacional Autónoma de México, Anthropos, Barcelona.

González Seguí, H. Oscar, 2003, "Reseña a A. Aguilera y A. López (2001)”, Educación Superior: Cifras y Hechos, año 
2, núm. 12, enero-febrero, Centro de Investigaciones Interdisciplinarias en Ciencias y Humanidades (CEIICH), Universidad Nacional Autónoma de México, pp. 13-14.

__ en prensa, "Entre modelos y discursos: complejidad y antropología”, Anales de Antropología, vol. 40, núm. 2, Instituto de Investigaciones Antropológicas, Universidad Nacional Autónoma de México, México.

Grimshaw, Anna y Keith Hart, 1994, "Anthropology and the Crisis of the Intelectuals", Critique of Anthropology, vol. 14, núm. 3, pp. 27-261

Grupo Antropocaos: Diego Díaz, Jordán Kristoff, Mora Castro, Jorge Miceli, Damián Castro, Ramón Quinteros, Sergio Guerrero, 2006, Exploraciones en antropología de la complejidad, Sb Editorial, Buenos Aires.

Gumerman, G. y M. Gell-Mann (eds.), 1994, Understanding Complexity in the Prehistoric Southwest, Addison-Wesley, SFI, Reading, Massachusetts.

Hacking, Ian, 2001, ¿La construcción social de qué?, Paidós Ibérica, Madrid.

Hannerz, Ulf, 1980, Exploring the City, Columbia University Press, Nueva York. [Trad. al español: Exploración de la ciudad, Fondo de Cultura Económica, México, 1986.]

Heylighen, F. y D. T. Campbell, 1995, "Selection of Organization at the Social Level: Obstacles and Facilitators of Metasystem Transitions", The Journal of General Evolution, núm. 45, pp. 181-212.

Hogg, Michael A., 2006, "Social Identity Theory", en Peter Burke (ed.), Contemporary Social Psychological Theories, Stanford University Press, Stanford, pp. 111-136.

Holland, John H., 1995, Hidden Order: How Adaptation Builds Complexity, Perseus Books, Cambridge, Massachusetts.

Hong, Y., M. Morris, C. Y. Chiu y V. Benet-Martínez, 2000, "Multicultural Minds: A Dynamic Constructivist Approach to Culture and Cognition", American Psychologist, núm. 55, pp. 709-720.

Horgan, John, 1995, "From Complexity to Perplexity", Scientific American, junio, pp. 74-79

Huberman, B. y N. S. Glance, 1993, "Diversity and Collective Action”, en H. Haken y A. Mikhailov (eds.), Interdisciplinary Approaches to Non Linearcomplex Systems, Springer, Verlag, Berlín, pp. 44-64.

Ibarra Colado, Eduardo, 2000, "Evaluación, productividad y conocimiento: barreras institucionales al desarrollo académico", Sociológica, vol. 14, núm. 41, pp. 41-59.

Jones, Doug, 1999, "Evolutionary Psychology”, Annual Review of Anthropology, núm. 28, pp. 553-575.

Johnson, Steven, 2003, Sistemas emergentes: o qué tienen en común hormigas, neuronas, ciudades y software, Turner, Fondo de Cultura Económica, Madrid.
Kauffman, Stuart, 1993, The Origins of Order. Self-organization and Selection in Evolution, Oxford University Press, Nueva York.

Kauffman, Stuart, 1995, At Home in the Universe. The Search for the Laws of Self-organization and Complexity, Oxford University Press, Nueva York.

Kitcher, Philip, 2001, El avance de la ciencia, Instituto de Investigaciones Filosóficas, Universidad Nacional Autónoma de México, México.

Knorr-Cetina, K. D., 1981, The Manufacture of Knowledge. An Essay on the Constructivist and Contextual Nature of Science, Pergamon, Oxford.

Lansing, J. Stephen, 2003, “Complex Adaptive Systems”, Annual Review of Anthropology, núm. 32, pp. 183-204.

Latour, Bruno, 2003, Science in Action. How to Follow Scientists and Engineers through Society, Harvard University Press, Cambridge, Massachusetts.

Lee, Richard E., 2000, "The Structures of Knowledge and the Future of the Social Sciences: Two Postulates, Two Propositions and a Closing Remark", Journal of WorldSystems Research, vol. VI, núm. 3, pp. 786-796.

Mansilla, R. y E. Ruelas (eds.), 2005, Las ciencias de la complejidad y la innovación médica, Universidad Nacional Autónoma de México, Plaza y Valdez, México.

Marcus, Joyce y Kent Flannery, 1996, Zapotec Civilization. How Urban Society Evolved in Mexico's Oaxaca Valley, Thames and Hudson, Londres.

Martínez Alier, Joan y Klaus Schlüpmann, 1991, La ecología y la economía, Fondo de Cultura Económica, México.

Maturana, Humberto y Francisco Varela, 1996, El árbol del conocimiento, Debate, Madrid.

Miramontes, Octavio, 1999, "Los sistemas complejos como instrumentos de conocimiento y transformación del mundo", en Santiago Ramírez (coord.), Perspectivas en las teorías de sistemas, Centro de Investigaciones Interdisciplinarias en Ciencias y Humanidades (CEIICH), Universidad Nacional Autónoma de México, Siglo XXI, México, pp. 83-92.

Mithen, Steven, 2006, "Ethnobiology and the Evolution of the Human Mind", Journal of the Royal Anthropological Institute (N.S.), S45-S61.

Molina, Virginia, 2005, "La complejidad: una propuesta reciente para el análisis del cambio", en Rosario Esteinou y Magdalena Barros (eds.), Análisis del cambio sociocultural, Centro de Investigaciones y Estudios Superiores en Antropología Social, México, pp. 71-102.

Morin, Edgar, 1981-1992, El método, 4 ts., Cátedra, Madrid. _, 2003 , Introducción al pensamiento complejo, Gedisa, Barcelona.

Palerm, Ángel, 1980, Antropología y marxismo, Centro de 
Investigaciones y Estudios Superiores en Antropología Social, Nueva Imagen, México.

Palerm Viqueira, Juan Vicente, 2000, “Las nuevas comunidades mexicanas en los espacios rurales de Estados Unidos, a propósito de una reflexión acerca del quehacer antropológico", en Virginia García Acosta, (coord.), La diversidad intelectual. Ángel Palerm, in memoriam, Centro de Investigaciones y Estudios Superiores en Antropología Social, México, pp. 63-112.

Pérez-Taylor, Rafael (comp.), 2002, Antropología y complejidad, Gedisa, Barcelona.

- (comp.), 2006, Anthropologías: avances en la complejidad humana, Sb Editorial, Buenos Aires.

Portugali, Juval, 1999, Self-organization and the City, Springer-Verlag, Berlín.

Prigogine, Ilya, 1996, El fin de las certidumbres, Andrés Bello, Santiago de Chile.

_ e Isabelle Stengers, 1983, La nueva alianza. Metamorfosis de la ciencia, Alianza, Madrid.

Ramos-Fernández, G., J. L. Mateos, O. Miramontes, G. Cocho, H. Larralde y B. Ayala Orozco, 2004, "Levy Walk Patterns in the Foraging Movements of Spider Monkeys (Ateles geoffroyi)", Behavioral Ecology and Sociobiology, vol. 55, núm. 2, pp. 224-230.

Reynolds, Robert, 1999, "An Overview of Cultural Algorithms", en Advances in Evolutionary Computation, Mac Graw Hill.

Reynoso, Carlos, 2006, Complejidad y caos: una exploración antropológica, Sb Editorial, Buenos Aires.

Roth, Andrew, 2008, "Impacto social y cultura nacional", ponencia presentada en el Seminario Impacto de las Ciencias Sociales, 15 de enero, El Colegio de Michoacán, Zamora.

Shore, Chris y Susan Wright, 1999, "Audit Culture and Anthropology: Neo-liberalism in British Higher Education”, Journal of the Royal Anthropological Institute, núm. 5 , pp. 557-575.

Snow, C. P., 1964, The Two Cultures and a Second Look, Cambridge University Press. (Trad. al español: Las dos culturas, Nueva Visión, Buenos Aires, 2000.)
Smith, Thomas S. y Gregory T. Stevens, 2002, "Hyperstructures and the Biology of Interpersonal Dependence: Rethinking Reciprocity and Altruism", Sociological Theory, vol. 20, núm. 1, marzo, pp. 106-130.

Sotolongo Codina, Pedro L. y Carlos J. Delgado Díaz, 2006, La revolución contemporánea del saber y la complejidad social, Consejo Latinoamericano de Ciencias Sociales, Buenos Aires.

Stetz, Jan, 2006, “Identity Theory”, en Peter Burke (ed.), Contemporary Social Psychological Theories, Stanford University Press, Stanford, pp. 88-110.

Suchman, Lucy, 2006, Human-Machine Reconfigurations. Plans and Situated Actions, Cambridge University Press, Cambridge, Massachusetts.

Thrift, Nigel, 1999, “The Place of Complexity”, Theory, Culture and Society, vol. 16, núm. 3, pp. 31-69.

Tilly, Charles, 1997, Roads from Past to Future, Rowman \& Littlefield Publishers, Lanham, Massachusetts.

Tomasello, Michael, 1999, "The Human Adaptation for Culture”, Annual Review of Psychology, núm. 28, pp. 509529.

Tooby, John y Leda Cosmides, 1997, Evolutionary Psychology: A Primer, en línea: < http://www.psych.ucsb.edu/ research/cep/primer.html $>$ (consultado en febrero de 2008).

Tyrtania, Leonardo, 1999, Termodinámica de la supervivencia de las ciencias sociales, Universidad Autónoma Metropolitana, México.

Varela, Roberto, 1984, Expansión de sistemas y relaciones de poder, Universidad Autónoma Metropolitana-Iztapalapa, México.

Wallerstein, Immanuel, 1996, Abrir las ciencias sociales: informe de la Comisión Gulbenkian para la reestructuración de las ciencias sociales, Siglo XXI, Universidad Nacional Autónoma de México, México.

Wolf, Eric R., 1986, “Cultura e ideología”, en Susana Glantz (comp.), La heterodoxia recuperada. En torno a Ángel Palerm, Fondo de Cultura Económica, México, pp. 582596. 